\section{$\underset{\substack{\text { hommes } \\ \text { \& migrations }}}{ }$}

\section{Hommes \& migrations}

Revue française de référence sur les dynamiques

migratoires

\section{$1299 \mid 2012$}

Musulmanes et féministes en Grande-Bretagne

\title{
Le débat français sur la burqa trouve un écho en Grande-Bretagne
}

\section{Khursheed Wadia}

Traducteur : Françoise Bouillot

\section{(2) OpenEdition}

\section{Journals}

Édition électronique

URL : http://journals.openedition.org/hommesmigrations/1409

DOI : 10.4000/hommesmigrations. 1409

ISSN : 2262-3353

Éditeur

Musée national de l'histoire de l'immigration

Édition imprimée

Date de publication : 1 septembre 2012

Pagination : 66-75

ISSN : 1142-852X

Référence électronique

Khursheed Wadia, "Le débat français sur la burqa trouve un écho en Grande-Bretagne », Hommes \& migrations [En ligne], 1299 | 2012, mis en ligne le 31 décembre 2014, consulté le 19 avril 2019. URL : http://journals.openedition.org/hommesmigrations/1409; DOI : 10.4000/hommesmigrations. 1409 


\section{Le débat français sur la burqa trouve un écho en Grande-Bretagne}

Par Khursheed Wadia, principal research fellow, université de Warwick

L'interdiction, votée par le Parlement français le 14 septembre 2011, du port du voile intégral dans les lieux publics a suscité de nombreuses réactions outre-Manche. Comme en France, cette loi met en évidence le clivage entre les partisans et les opposants d'une intervention du législateur dans un domaine où se croisent choix privé, vie intime et respect de l'ordre public. Si une telle mesure n'est pas à l'ordre du jour en Angleterre, deux militantes musulmanes donnent leur avis sur le débat. 
Les controverses françaises sur la tenue des femmes musulmanes et les interdictions prononcées d'abord contre le hijab (foulard) dans les écoles publiques (2004) et plus récemment contre la burqa (voile intégral) dans les lieux publics (2011) ont trouvé un écho de l'autre côté de la Manche et suscité un débat politique en Grande-Bretagne.

\section{Le contexte politique britannique}

Comme en France, cette interdiction a divisé l'opinion publique en Grande-Bretagne. D'une part, l'État français a été applaudi pour avoir assuré une prétendue 'juste défense des valeurs françaises et européennes contre une idéologie et une pratique religieuse étrangères' non seulement par des politiciens et des journalistes populistes, mais aussi par des groupes d'intellectuels (universitaires, journalistes et sociologues) persuadés que l'interdiction allait protéger les femmes que leur famille et leur communauté contraignaient à porter le voile, et faire ainsi respecter les droits des femmes. D'autres adoptaient un point de vue pragmatique selon lequel l'interdiction de la burqa allait empêcher les terroristes d'échapper aux contrôles de sécurité et faciliter le travail des services lorsqu'il est crucial de voir le visage de son interlocuteur.

Par ailleurs, les points de vue suivants se sont opposés à l'interdiction sans qu'ils soient nécessairement liés à la gauche ou à la droite, aux musulmans ou aux non-musulmans : le droit fondamental à l'expression religieuse est bafoué ; il est répréhensible de se ranger aux côtés d'un État tout-puissant, muni de tous les moyens de coercition, contre un petit groupe dépourvu de pouvoir ; la burqa n'est pas la cause, mais le symbole de l'inégalité et de la soumission ; en interdisant la burqa, l'État français adopte une attitude comparable à celle d'États absolutistes comme l'Arabie saoudite, où les femmes sont contraintes de dissimuler leur visage. Le débat sur l'interdiction de la burqa, paru dans The Guardian le 2 avril 2011, entre deux figures bien connues de la vie publique britannique ${ }^{(1)}$,Yasmin Alibhai-Brown et Salma Yaqoob, illustre la perception que les Britanniques ont du traitement de l'islam dans la vie politique française.

\section{Trajectoires de militantes}

Yasmin Alibhai-Brown est actuellement journaliste et animatrice de radiotélévision. Au cours de ces vingt dernières années, elle a écrit régulièrement pour la presse quotidienne et périodique de Grande-Bretagne (notamment The Guardian, The Independent et The Observer) et des États-Unis (The New York Times, Time et Newsweek). Elle a, en outre, réalisé des émissions pour plusieurs chaînes de radio 
et de télévision, dont la BBC et Channel Four. Elle a publié de nombreux ouvrages $^{(2)}$. Elle a obtenu de nombreux prix, dont, en 2002, le prix George Orwell du journalisme politique.

Yasmin Alibhai-Brown est née et a grandi en Ouganda. Elle est arrivée en GrandeBretagne en 1972, juste avant l'expulsion des communautés sud-asiatiques d'Ouganda par le gouvernement d'Idi Amin Dada. Elle a d'abord étudié la littérature anglaise à Oxford, où elle a obtenu un MPhil (l'équivalent du DEA). Elle s'est lancée dans une carrière de journaliste vers l'âge de trente ans, après plusieurs années d'enseignement, notamment auprès des populations réfugiées et immigrées. Dans les années quatre-vingt-dix, elle a aussi été chercheure à l'Institute for Public Policy Research, un groupe de réflexion proche du Parti travailliste. Elle est toujours attachée au Foreign Policy Centre ${ }^{(3)}$ en tant que maître de recherche.

Se décrivant elle-même comme "libérale de gauche, antiraciste, féministe et musulmane", Yasmina Alibhai-Brown n'est pas restée étrangère à la controverse, s'attirant de violentes critiques de la part de la droite comme de la gauche britanniques. On l'a accusée de soutenir des positions contradictoires et/ou hypocrites sur un certain nombre de questions, bien qu'elle ait revendiqué le droit de changer d'avis et d'éprouver de l'empathie pour les deux protagonistes d'un conflit. Elle a ainsi affirmé sa conviction que l'État d'Israël offrait un réel sentiment de sécurité aux juifs, tout en défendant les droits des Palestiniens qui sont récusés par ce même État israélien. Plus récemment, elle a encouru un désaveu public sans appel en déclarant sur une radio de la BBC, qu'aucun politicien britannique n'avait le droit moral de commenter les violations des droits de l'homme à l'étranger, y compris la lapidation des femmes en Iran. La controverse s'est ensuite poursuivie quand un politicien de droite a suggéré qu'elle devait être elle-même lapidée à mort ${ }^{(4)}$. Sur la burqa, Alibhai-Brown s'est attiré les foudres de musulmans qui l'accusent de trahir sa communauté et de saper les droits de celle-ci dans un climat hostile aux musulmans et à l'islam. Elle a toutefois maintenu une position fermement républicaine, en soutenant qu'au bout du compte, aucune culture ou tradition, qu'elle soit religieuse ou autre, ne doit être à l'abri des critiques pour l'unique raison qu'elle représente la tradition.

Salma Yaqoob était jusqu'en septembre 2012 à la tête du Respect Party, créé en 2004 à partir de la conviction qu'une claire alternative de gauche était nécessaire face aux trois principaux partis de gouvernement (le Parti travailliste, le Parti conservateur et les Démocrates libéraux), qui avaient entraîné la Grande-Bretagne dans la guerre d'Irak malgré les protestations véhémentes de millions de gens ordinaires. ${ }^{(5)}$

Le Respect Party a connu un parcours chaotique, bien qu'il ait compté plusieurs figures politiques et publiques d'importance dans ses rangs, notamment George Monbiot (militant pour l'environnement et écrivain), Ken Loach (réalisateur) et 
surtout George Galloway, ancien député travailliste de Glasgow qui a été exclu du Parti travailliste en 2003 du fait de son opposition résolue à la guerre en Irak ${ }^{(6)}$.

Si le Respect Party est le parti de la gauche radicale qui a connu le plus grand succès en Grande-Bretagne ${ }^{(7)}$, il a été affaibli par des luttes de tendances, surtout entre 2007 et $2009^{(8)}$. Il a toutefois connu récemment un regain de popularité dans le nord de l'Angleterre où les politiques d'austérité du gouvernement de Cameron ont eu un impact dévastateur sur les populations les plus défavorisées, tandis que le Parti travailliste renonçait à défendre ses fiefs ouvriers traditionnels dans le Nord et ailleurs contre ces mêmes politiques. En mars 2012, George Galloway a donc remporté le siège de député de Bradford West par une victoire spectaculaire sur le candidat du Parti travailliste ${ }^{(9)}$.

Bradford-Ouest se caractérise par une forte implantation ouvrière et une importante population musulmane. Ce résultat s'est confirmé aux élections locales de mai 2012, qui ont donné au Respect Party 5 sièges au conseil municipal de BradfordOuest $^{(10)}$.

Si le Respect Party a connu des fortunes diverses au cours de ces cinq dernières années, Salma Yaqoob n'a cessé de voir s'accroître sa popularité dans sa ville natale

Le débat sur l'interdiction
de la burqa, paru dans
The Guardian le 2 avril 2011 ,
entre deux figures bien
connues de la vie publique
britannique, Yasmin Alibhai-
Brown et Salma Yaqoob,
illustre la perception
que les Britanniques ont
du traitement de l'islam dans
la vie politique française.

de Birmingham, tout en gagnant en

crédibilité dans les grands médias et dans les milieux politiques. Elle a écrit régulièrement dans la presse depuis 2004, notamment au Guardian et au New York Times. Elle est devenue une figure familière de la radio et de la télévision britanniques, faisant l'objet de nombreux articles. Elle est souvent consultée sur l'Afghanistan, l'Irak, le conflit israélo-palestinien, l'immigration, le racisme, le multiculturalisme britannique et les questions d'égalité et de justice.

Née à Bradford, dans le nord de l'Angleterre, Salma Yaqoob a grandi à Birmingham. Elle a étudié la biochimie et la psychologie à l'université avant de devenir psychothérapeute. Elle a interrompu une thèse de doctorat quand elle s'est engagée dans la politique. C'est le jour où un homme a craché sur elle quelques jours après les événements du 11 septembre 2001 qu'elle a décidé de militer ${ }^{(11)}$. Elle a compris alors que les musulmans de Grande-Bretagne et d'ailleurs allaient devoir payer pour les atrocités qui avaient eu lieu à New York et à Washington, et qu'il fallait contrer l'hostilité publique avant qu'elle ne se déploie en un conflit social ouvert. Lors de l'invasion de l'Afghanistan, moins d'un mois après le 11 septembre, elle a organisé 
des manifestations contre la guerre. Son activisme n'a cessé depuis de s'intensifier, et en 2004 elle est devenue vice-présidente du Respect Party et leader de la Stop the War Coalition de Birmingham.

L'année 2005 a constitué un nouveau tournant pour elle et pour les musulmans de Grande-Bretagne en général ; c'est l'année où de jeunes musulmans britannique nés en Grande-Bretagne ont commis les attentats meurtriers du métro de Londres. Elle a alors travaillé à établir des passerelles entre les musulmans, les juifs et les chrétiens britanniques pour tenter de panser les blessures causées par les événements du mois de juillet; elle a reçu des messages haineux et des menaces de mort tout à la fois de la part de groupes racistes et de groupes islamistes extrémistes ; cette même année, elle s'est présentée aux élections législatives, arrivant en deuxième position avec 27,5\% des voix ${ }^{(12)}$. En 2006, Salma Yaqoob a été élue au conseil municipal de Birmingham, avec l'une des majorités les plus solides de l'histoire de la ville, mais elle a dû abandonner ce poste à l'automne 2011 pour des raisons de santé.

En tant que leader de la Stop the War Coalition de Birmingham et, jusqu'en septembre 2012, comme leader du Respect Party, elle continue de poursuivre sa campagne pour la paix et pour l'égalité des droits des populations défavorisées en Grande-Bretagne et ailleurs.

\section{"La France a-t-elle raison d'interdire la burqa ?" (The Guardian, 2011)}

Depuis le 11 avril 2011, les femmes en France tombent sous l'interdiction de porter le niqab - le voile intégral - dans les lieux publics. Amelia Hill a mis face à face deux musulmanes britanniques de premier plan pour discuter de cette initiative controversée. Salma Yaqoob, leader du Respect Party, y est opposée, alors que pour Yasmina AlibhaiBrown, les Français ont peut-être pris la bonne décision.

Yasmin Alibhai-Brown : Je ne suis pas du tout d'accord avec [le voile intégral] et je suis alarmée par la vitesse à laquelle il se répand. II y a cinq ans, les gens me disaient que seules quelques femmes le portaient. Aujourd'hui, c'est une épidémie.

Salma Yaqoob : Je différencie mes réactions personnelles et émotionnelles du droit des autres à prendre leurs propres décisions. Personnellement, je n'aime pas le voile intégral. Je peux comprendre pourquoi il met des gens mal à l'aise. Mais je me sens encore plus mal à l'aise devant l'interdiction de ce choix. Ma mère ne portait pas le hijab 
(foulard) et elle n'était pas d'accord quand j'ai commencé à le faire. Vers l'âge de vingt ans, ma sœur a commencé à porter une burqa intégrale. Cela me mettait très mal à l'aise, mais elle la concevait comme une expression de sa spiritualité. À présent, elle ne la porte plus. Ce n'était qu'un moment de son itinéraire. Ce qui est fondamental pour moi, c'est que personne ne devrait dicter à quiconque ce qu'il doit couvrir ou ne pas couvrir.

Y. A.-B. : C'est ce relativisme qui me pose problème dans ce que vous dites. La foi ne demande pas cela. C'est un projet wahhabite, financé par les Saoudiens, qui a réussi à s'implanter ici. Je ne veux pas interdire le voile à la façon des Français. Les Français sont racistes. Ils le font pour de mauvaises raisons. Mais ils ont pris la bonne décision. En Grande-Bretagne, je crois vraiment que nous avons le droit de dire que dans les espaces publics et les métiers publics, ce choix n'est pas un choix. Nous ne pouvons pas tous choisir de porter ce que nous voulons.

S. Y. : Ce que vous dites est très dangereux et antilibéral. Cette discussion est étouffée parce qu'elle impose des interprétations qui mettent les gens sur la défensive. Ce n'est pas une conversation décontextualisée.

Y. A.-B. : Vous avez raison : elle n'est pas décontextualisée. Pendant dix ans, j'ai même pris position contre le hijab parce qu'il dit quelque chose sur ce que sont les femmes et sur ce que font les femmes. Nous devrions réfléchir aux significations qu'il porte.

S. Y. : Mais, Yasmin, vous dites : "Je n'aime pas ça, donc je vais vous refuser le droit de le faire." Que trouvons-nous de si précieux dans notre société ? La liberté qui nous permet de porter ce que nous voulons. Je pense qu'il est très paternaliste - et même antiféministe - de priver quelqu'un de ce choix, ou de dire qu'une femme n'est acceptable pour vous que si elle est habillée d'une façon qui vous convient.

Y. A.-B. : Mais le voile a acquis de telles connotations. Il dit que les cheveux, le visage ou le corps d'une femme, si on les regarde, mettent sa pudeur en danger. C'est une véritable insulte pour les hommes : qu'est-ce que cela nous dit sur eux? Et le voile dit aussi que vous vous préservez pour un homme. Que vous ne vous déballez que pour lui. Ce sont là des implications graves pour les féministes. La question n'est pas que je dise, moi : "Ça ne me plait pas." II s'agit de dire, en tant que féministe, que je ne supporte pas le message sous-jacent que "la femme, c'est le diable".

S. Y. : Vous projetez cette interprétation extrême sur tout le monde. Si c'est la réaction que le voile suscite chez vous, vous devez admettre qu'elle puisse être totalement différente chez d'autres. Quand j'ai commencé à porter le hijab, ça n'avait rien à voir 
avec tout ça. Mon problème, c'était de refuser d'être jugée sur mon apparence. En imposant sur ce point les lois de l'État, vous imposez une seule interprétation et vous refusez la liberté d'en proposer d'autres. Ce qui m'inquiète, c'est l'obsession que suscite le voile des deux côtés de la controverse.

Y. A.-B. : C'est la sexualisation des femmes que je ne supporte pas ; l'idée que les femmes sont une sorte de présence démoniaque.

S. Y. : Oui, mais comment apporter de véritables changements et amener les gens à penser? Sûrement pas en leur imposant des lois qui mettent les femmes sur la défensive. L'émancipation des femmes a pu se faire parce que le discours de l'égalité a été intériorisé. Si vous fermez la porte à ce discours, les gens deviennent hyperdéfensifs.

Y. A.-B. : J'accepte totalement ce que vous dites. Et si nous disions : dans la vie publique, au travail et dans les lieux publics, on doit voir notre visage ? Cet obstacle aux échanges humains provoque un apartheid entre certaines femmes musulmanes et toutes les autres femmes. Cela transmet le message implicite qu'on est contaminé. L'autre jour, on a célébré l'Aïd pour la première fois près de chez moi à Ealing Common. Je me suis dit que c'était fantastique, et j'ai voulu y participer, mais on m'a repoussée parce que je n'étais pas voilée.

S. Y. : Et vous vous êtes sentie comment?

Y. A.-B. : J'étais furieuse. Ils n'ont pas le droit de m'exclure.

S. $\mathbf{Y}$ : : Et si vous aviez la tête couverte et qu'on vous disait le contraire ? Vous exclure ainsi aurait-il nourri le dialogue ? Etes-vous partie en pensant : "S'ils m'ont exclue, c'est que je dois faire quelque chose de mal" ? Une femme à qui on dit qu'elle ne peut pas porter quelque chose ne risque pas de se sentir encouragée à prendre part au dialogue.

Y. A.-B. : Si vous avez dialogué, pourquoi cette épidémie ne s'est-elle pas arrêtée?

S. Y. : N'utilisez pas ce mot-là, c'est dangereux.

Y. A.-B. : Je suis désolée, mais pour moi c'est une épidémie. La génération de ma mère a voulu une religion qui lui donnait des libertés personnelles. Elles ont jeté le voile et maintenant le voilà qui revient. II y a du lavage de cerveau dans l'air, et nous sommes assises là en train de parler de choix. 
S. Y. : Venez me voir à Birmingham, Yasmin, et vous verrez le choix. Vous verrez des femmes qui portent le voile, des femmes qui le portaient et ne le portent plus, des femmes qui ne l'ont jamais porté mais le feront peut-être à l' avenir. II ne fait pas de doute que certaines ont subi des pressions de leur famille, mais elles vivent pour la plupart dans une société hostile où le voile n'est pas le bienvenu. Porter le voile n'est pas une chose facile dans la société d'aujourd'hui. L'idée que celles qui le portent sont des victimes passives de la famille ou adoptent une posture anti-occidentale est fausse. C'est seulement quand la société devient moins tolérante que cela devient un problème.

Y. A.-B. : Pourquoi la société devrait-elle être tolérante vis-à-vis d'un signe disant que les femmes sont des tentatrices diaboliques ou des paquets dont la sexualité doit être contrôlée?

S. $\mathbf{Y}$ : : On peut aussi l'interpréter comme une affirmation dans l'autre sens. Certaines femmes trouvent qu'il est libérateur et émancipateur de se découvrir tandis que d'autres jugent cela opprimant. Une interdiction revient à dire aux musulmans qu'ils ne sont pas d'ici. Cela n'encourage pas un sentiment d'appartenance fondé sur des relations multiculturelles. C'est tout le contraire. Ce n'est qu'une forme à peine voilée de condescendance et de racisme. Ce n'est pas émanciper les femmes. C'est faire exactement le contraire. Cela n'encourage pas la cohésion ni le respect - cela encourage la peur.

Y. A.-B. : Ne me parlez pas d'appartenance. Aucune des filles musulmanes de la classe de ma fille ne refuserait de venir à son anniversaire parce que je ne porte pas le voile. Il y a une autoségrégation en cours et ce bout de tissu en est le symbole.

Amelia Hill : Cette interdiction est-elle l'expression d'un cynisme politique ou une authentique préoccupation sur le type de société et de modernité que nous voulons?

S. $\mathbf{Y}$ : : Je pense que les politiciens français ne sont pas ingénus. Ils cherchent à ratisser l'électorat de droite avec ce type de rhétorique. C'est bien une affaire de cynisme politique. Je pense que la force de ce pays [le Royaume-Uni] est qu'il permet un authentique dialogue sans interdictions brutales. [En France] ce n'est pas un débat entre égaux : cette interdiction va achever d'isoler les gens. Ce n'est pas créer une citoyenneté engagée, c'est le contraire même : décourager les gens et leur ôter tout espoir. 


\section{La Grande-Bretagne sur les pas de la France?}

Cette conversation reflète les arguments entendus en Grande-Bretagne pour et contre le voile islamique, arguments maintes fois repris au cours de ces dix dernières années. Les "débats sur le voile" propres aux Britanniques ont eu lieu au milieu des années deux mille. En 2005, une lycéenne de 16 ans, Shabina Begum, a intenté un procès pour défendre son droit à porter le jilbab (le corps et la tête étant complètement couverts mais pas le visage) en infraction avec la politique de son école, qui l'interdisait parce qu'il ne correspondait pas aux critères de l'uniforme. Elle a perdu devant la Haute Cour ${ }^{(13)}$, gagné en appel devant la Cour d'Appel ${ }^{(14)}$, mais a perdu en dernière instance devant la Chambre des Lords ${ }^{(15)}$. Le ministère de l'éducation avait espéré que ce jugement clarifierait la position des écoles sur ce que peuvent porter ou non les élèves, mais ce fut peine perdue, les juges ayant précisé que leur décision ne pouvait constituer un précédent, puisqu'elle se fondait sur un cas particulier.

Le second "débat sur le voile" de Grande-Bretagne a eu lieu en octobre 2006, quand Jack Straw, alors ministre travailliste des Affaires étrangères, a déclaré à son journal local qu'il préférait parler à des femmes ne portant pas le niqab(voile intégral du visage) et qu'il les prierait de l'ôter si elles souhaitaient le consulter. Il expliqua ensuite qu'il avait voulu ouvrir le débat public sur le sujet, et qu'il était en faveur de l'abolition du voile "du fait de la séparation qu'il implique ${ }^{(16) "}$. Si l'intervention de Straw sur le voile islamique a provoqué la controverse et encouragé, selon certains, diverses agressions physiques contre des femmes portant le voile intégral, elle a eu un impact politique limité. En fait, en 2010, Straw a présenté des excuses publiques pour ses commentaires de 2006 : "Si javais mesuré la publicité qui serait donnée à mes propos en octobre 2006, je m’en serais abstenu et je suis désolé qu'ils aient provoqué autant de problèmes..."

À n'en pas douter, ces débats referont surface à l'avenir, mais vu la place accordée à la religion dans l'État britannique et dans les institutions de la société civile (voir l'article ci-dessus dans ce numéro sur la place des musulmans dans la société britannique), il est peu probable que la Grande-Bretagne connaisse une interdiction du voile comme celle de la France.

\section{Traduit de l'anglais par Françoise Bouillot}

\section{Notes}

1. Nous remercions The Guardian qui a donné la permission à Hommes \& Migrations de publier l'article intitulé "Is France right to 'ban the burqa' ?", d'Amelia Hill, dans sa totalité (Licence No R22X73). L'article original peut être consulté sur le site www.guardian.co.uk/commentisfree/2011/apr/02/conversation-yaqoob-alibhai-brown-burqa. 
2. Parmi les ouvrages deYasmin Alibhai-Brown, voir notamment, No Place Like Home, Londres, Virago Press Ltd, 1995 ; Some of My Best Friends Are..., London, Politico's Publishing House, 2004 ; The Settler's Cookbook: a Memoir of Love, Migration and Food, London, Portobello Books, 2009; Who Do We Think We Are? Imagining the New Britain, Londres, Allen Lane, 2000.

3. Groupe de réflexion centriste, le FPC a été fondé en 1998 par des membres du Parti travailliste.

4. Caroline Gammell, "Tory Councillor Gareth Compton Arrested over Twitter 'Stoning' Tweet about Yasmin AlibhaiBrown", in The Telegraph, 11 novembre 2010.

www.telegraph.co.uk/news/politics/conservative/8127081/Tory-councillor-Gareth-Compton-arrested-over-Twitterstoning-tweet-about-Yasmin-Alibai-Brown.html.

5. Le Respect Party se positionne dans la gauche radicale et a été fondé sur les principes de la paix, de la propriété des services publics par l'État et le plein emploi. Son nom est un acronyme de Respect, Equality, Socialism, Peace, Environmentalism, Community et Trade Unionism.

6. Les lecteurs intéressés peuvent consulter Timothy Peace, "All I'm Asking, is for a Little Respect: Assessing the Performance of Britain's Most Successful Radical Left Party", in Parliamentary Affairs, 2012,

http://pa.oxfordjournals.org/content/early/2012/01/12/pa.gsr064.full.pdf+html);

Farzana Schain, "Uneasy Alliances: British Muslims and Socialists Since the 1950s", in Journal of Communist Studies and Transition Politics, vol. 25, $\mathrm{n}^{\circ}$ 4, 2009, pp. 95-109.

7. Timothy Peace, op. cit.

8. Farzana Schain, op. cit.

9. Patrick Wintour, "George Galloway Wins Bradford West byelection”, in The Guardian, 30 mars 2012. www.guardian.co.uk/politics/2012/mar/30/george-galloway-bradford-west-byelection.

10. Helen Pidd, "George Galloway's Respect Party Wins Bradford Council Seats", in The Guardian, 4 May 2012. www.guardian.co.uk/politics/2012/may/04/george-galloway-respect-bradford-council.

11. Salma Yaqoob, "Salma Yaqoob, 33", in The Guardian, 30 November 2004. www.guardian.co.uk/uk/2004/nov/30/ islamandbritain 16 .

12. Electoral Commission, "UK general election 2005. Results for Birmingham Sparkbrook \& Small Heath", 2005. www.electoralcommission.org.uk/elections/ results/general_elections/uk-general-election2005/birmingham-sparkbrook —and-small-heath.

13. $B B C$ News, "Schoolgirl loses Muslim gown case", BBC News, 15 June 2004.

http://news.bbc.co.uk/1/hi/education/ 3808073.stm.

14. $B B C$ News, "Schoolgirl Wins Muslim Gown Case", BBC News, 2 March 2005. http://news.bbc.co.uk/1/hi/england/ beds/bucks/herts/4310545.stm.

15. House of Lords, "Judgments - R (on the application of Begum (by her litigation friend, Rahman)) (Respondent) v. Headteacher and Governors of Denbigh High School (Appellants)", in UKHL 15, 2006. www.publications.parliament.uk/pa/ ld200506/ldjudgmt/jd060322/begum-1.htm.

16. BBC News, "Remove Full Veils' Urges Straw", BBC News, 6 October 2006.

http://news.bbc.co.uk/1/hi/uk/5411954.stm. 